\title{
Solid phase recrystallization of ZnS thin films on sapphire
}

\author{
Z. Z. Bandić, ${ }^{\text {a) }}$ E. C. Piquette, J. O. McCaldin, and T. C. McGill ${ }^{\text {b) }}$ \\ Thomas J. Watson, Sr. Laboratory of Applied Physics, California Institute of Technology, \\ Pasadena, California 91125
}

(Received 17 November 1997; accepted for publication 29 March 1998)

\begin{abstract}
High quality $\mathrm{ZnS}$ thin films are important for light emitting diodes based on $\mathrm{ZnS}$, which is a very efficient phosphor. To improve as grown, molecular beam epitaxial, (111)-oriented cubic ZnS films, where defects were introduced due to the large mismatch between $\mathrm{ZnS}$ and a sapphire substrate $(\sim 20 \%)$, the $\mathrm{ZnS}$ was recrystallized by annealing at temperatures in the $825-1000{ }^{\circ} \mathrm{C}$ range, and sulfur pressures of $10 \mathrm{~atm}$. The films have been structurally characterized by high-resolution $\mathrm{x}$-ray diffraction, and electron diffraction by electron channeling patterns. Structural properties of the films annealed at temperatures above $900^{\circ}$ have improved significantly. Tilting in the recrystallized films has been reduced more than tenfold, with the recrystallized grains being defect-free. Most films were recrystallized in the as-grown, cubic form, as shown by electron channeling patterns. The surfaces of the films have been inspected with scanning electron microscope, and on most samples they have been found to remain smooth, although on some of the films annealed at elevated temperatures we have observed hexagonal pits. The role of sulfur gas overpressure in the recrystallization has been discussed, and possible effects on film evaporation, grain boundary migration and compliancy of sapphire substrate have been analyzed. (C) 1998 American Institute of Physics. [S0003-6951(98)03022-8]
\end{abstract}

Zinc sulfide, with its wide band gap $(\sim 3.8 \mathrm{eV})$ and excellent luminescence, which can be obtained in almost the whole visible range with suitable dopants, is a very attractive material for light emitting diodes (LEDs), ${ }^{1,2}$ lasers, ${ }^{3}$ and flatpanel electroluminescent (EL) displays. ${ }^{4,5}$ We have recently proposed $^{6}$ and demonstrated ${ }^{7}$ an $n-\mathrm{ZnS}:(\mathrm{Ag}, \mathrm{Al}) / p-\mathrm{GaN}: \mathrm{Mg}$ heterostructure blue light emitting LED. The fabrication of this device requires growth of $\mathrm{ZnS}$ on a heavily mismatched GaN substrate. The initial phases of growth in the heavily mismatched interface geometry are the most critical, and it is not expected that the $\mathrm{ZnS}$ epilayer would grow coherently for more than 1 monolayer. $^{7}$ In fact, the large mismatchrelated energy will be relaxed in the form of tilting and threedimensional relaxation. ${ }^{7,8}$ This would certainly damage the overall crystalline quality of the $\mathrm{ZnS}$ layer, which is known to be critical to the performance of the II-VI-based optical devices. $^{9}$

In this study, we report on the solid phase recrystallization (SPR) of undoped, 0.3-0.5 $\mu \mathrm{m}$ thick, molecular beam epitaxy (MBE) grown, (111)-oriented, cubic $\mathrm{ZnS}$ films on sapphire ( $\sim 20 \%$ mismatch). Details about the growth system and procedures can be found in Ref. 7. Thermal annealing at large sulfur overpressure consistently improved the structural properties of the films, thereby compensating the effects of strong mismatch. Some of the previous efforts on recrystallization of thin films are described in Ref. 10. Presently, recrystallization is an important industrial process, and plays a vital role in the manufacture of steel and aluminum, ${ }^{11}$ and $\mathrm{HgCdTe} .{ }^{12}$ Solid phase recrystallization of bulk $\mathrm{ZnSe}$, CdTe and CdSe was recently successfully demonstrated by Triboulet et al., ${ }^{13,14}$ by annealing at the high temperatures

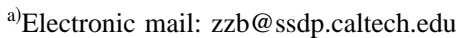

${ }^{b)}$ Electronic mail: tcm@ssdp.caltech.edu
}

under high column VI element overpressure, and excellent optical and structural properties were obtained. ${ }^{15}$

To contain $\mathrm{ZnS}$ samples and sulfur during the experiment, quartz ampoules with $2 \mathrm{~mm}$ thick walls were used. Prior to the experiment, the ampoules were cleaned with organic solvents (acetone and isopropyl alcohol) and then baked at $1000{ }^{\circ} \mathrm{C}$ for $2-4 \mathrm{~h}$. The MBE-grown undoped $\mathrm{ZnS} /$ sapphire films were placed in the ampoules together with 100-200 mg of $99.9999 \%$ sulfur. The amount of sulfur placed in the ampoule was calculated according to the van der Waals equation of state, so that gas pressure of $10 \mathrm{~atm}$ is reached on the anneal temperature. The ampoules were then attached to a turbo-molecular pump to attain a vacuum of $1 \times 10^{-6}$ Torr, and then sealed while the pump was still running. The sealed ampoules were then transferred into the temperature-controlled furnace. The temperature was slowly increased at the rate of $2-3{ }^{\circ} \mathrm{C} / \mathrm{min}$ to avoid thermal strains, until the final temperature in the $825-1000{ }^{\circ} \mathrm{C}$ range was achieved. After the samples were kept at this temperature for 2-20 h, the temperature was reduced slowly at the rate of $2-3{ }^{\circ} \mathrm{C} / \mathrm{min}$, again to avoid thermal stressing.

Samples were characterized before and after the annealing by ex situ high resolution x-ray diffraction (HRXRD), using a Philips PW3710 materials research diffractometer. The $\Omega$ and $\Omega / 2 \Theta$ scans around the ZnS thin film (111) reflections were performed in the four- or six-crystal mode (without or with a Bonse-Hart collimator in front of the detector), respectively, using $\mathrm{Ge}(220)$ reflections. To characterize the effects of different anneal temperatures, one sample (II391) was cut into five pieces, and each of them was annealed at different temperatures between 825 and $922^{\circ} \mathrm{C}$. The anneal temperature has to be chosen below the melting temperature, but has to be high enough so that the original grain boundaries become unstable. At that point, the 


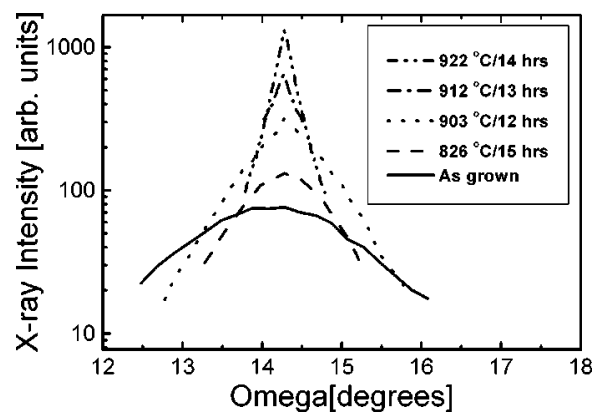

FIG. 1. Summary of x-ray $\Omega$ scans for different recrystallization temperatures of sample II391. The curves for samples recrystallized at higher temperatures have lower widths and higher maximum intensity.

conditions are favorable for the migration of grain boundaries and growth of defect-free nuclei. The anneal time ranged between 11 and $15 \mathrm{~h}$, and sulfur overpressure was kept constant at $10 \mathrm{~atm}$. The x-ray $\Omega$ scans (Fig. 1) and the full width at half maximum (FWHM) of the curves as a function of temperature (Fig. 2) indicate the amount of mosaic tilting present in the films, ${ }^{16}$ and show that the crystalline mosaicity is reduced significantly at anneal temperatures above $900{ }^{\circ} \mathrm{C}$. As-grown samples exhibited mosaic structure with grains approximately $10 \mu \mathrm{m}$ or less in size. Following recrystallization, the degree of mosaic tilting reduced approximately an order of magnitude, while the size of mutually tilted grains increased to approximately $130 \mu \mathrm{m}$ or less. However, the overall crystalline orientation was preserved across the grain boundaries, which was verified by $\mathrm{x}$-ray diffraction on the asymmetric set of crystalline planes. The typical FWHM of $\Omega / 2 \Theta$ curves for the samples in the II391 series after recrystallization was around 50 arcsec measured in the six-crystal configuration (using a Bonse-Hart monochromator with 12 arcsec acceptance angle in front of the detector). It was not possible to use this particular configuration before recrystallization, because the quality of the crystal was not good enough. Measured with the four-crystal configuration, the typical width before recrystallization was around $270 \operatorname{arcsec}$ (450 arcsec was typically measured, but there is a correction of 180 arcsec for the slit width). The width of the $\Omega / 2 \Theta$ scan corresponds to the crystalline quality of the individual grains, and in the case of the symmetrical reflection [like (111)] it depends on the thickness $L$ of the sample approximately as $\sim 0.886 \lambda /(\cos \theta L) \sim 29^{\prime \prime} / L(\mu \mathrm{m}) .{ }^{17}$ Since the thickness of the sample II391 was $0.45 \mu \mathrm{m}$, the width of 50 arcsec corresponds to recrystallized, defect-free

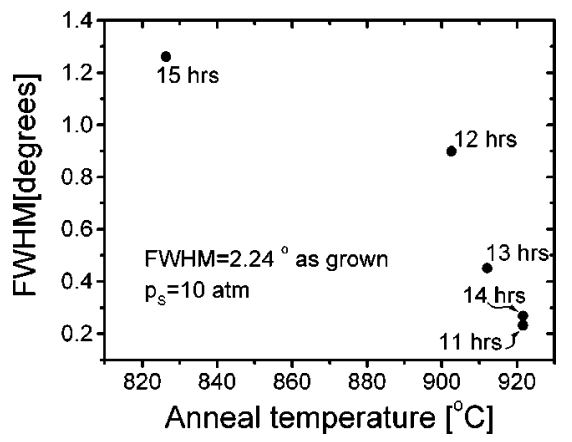

FIG. 2. Summary of FWHMs of x-ray $\Omega$ scans for different recrystallization

initial curve, so that curve widths can be compared.
temperatures of sample II391.
Downloaded 21 Dec 2005 to 131.215 .225 .171 . Redistribution subject to AlP license or copyright, see http://apl.aip.org

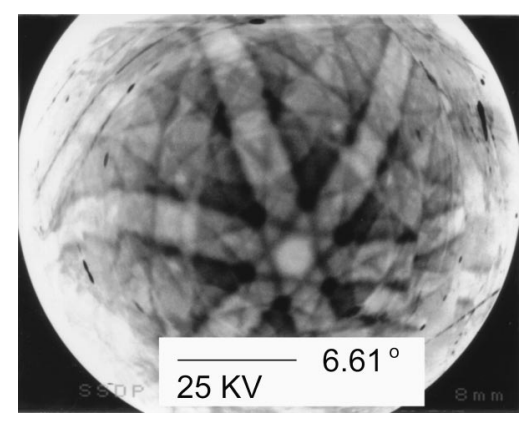

FIG. 3. Electron channeling pattern of the sample II366, recrystallized at $970{ }^{\circ} \mathrm{C}$ for $3 \mathrm{~h}$ at sulfur pressure of $10 \mathrm{~atm}$. The threefold symmetry of the pattern indicates cubic crystalline lattice and that no phase transition occurred. The fine structure in the pattern confirms good crystallinity of the sample.

grains. Electron channeling patterns (ECPs) were obtained in a JEOL $6400 \mathrm{~V}$ scanning electron microscope (SEM). The pattern for sample II366, recrystallized at $970{ }^{\circ} \mathrm{C}$ for $3 \mathrm{~h}$ at sulfur pressure of $10 \mathrm{~atm}$, is shown in Fig. 3. The corresponding $\Omega$ and $\Omega / 2 \Theta$ scans, before and after recrystallization, are shown in Fig. 4. It was not possible to obtain visible ECPs for samples before they were recrystallized, probably due to tilting and the large number of defects. In Fig. 3 we notice fine structure, where each line corresponds to the set of crystalline planes reflecting or absorbing high energy electrons. The high level of detail on this image corresponds to good crystallinity, in agreement with the x-ray measurements. The pattern has three-fold symmetry, indicating that the sample is indeed cubic, and that no phase transition to the hexagonal crystal modification (which would have six-fold symmetry) occurred.

The changes in surface morphology of the recrystallized samples have been investigated with the SEM. On most of the samples, the surface morphology after recrystallization remained smooth, which is important for device-related reasons, like electrical contacting. However, on some samples recrystallized at higher temperatures we observed hexagonal pits (see Fig. 5). One possible reason for the formation of pits at higher recrystallization temperatures is excess crystalline volume associated with a large density of defects. During the recrystallization process and boundary migration, the annihilation of defects takes place, during which volume of the crystal reduces, resulting in the pit formation. At lower temperatures, however, the boundary migration is slower and crystal relaxation times larger, which leads to fewer or no pits formed.
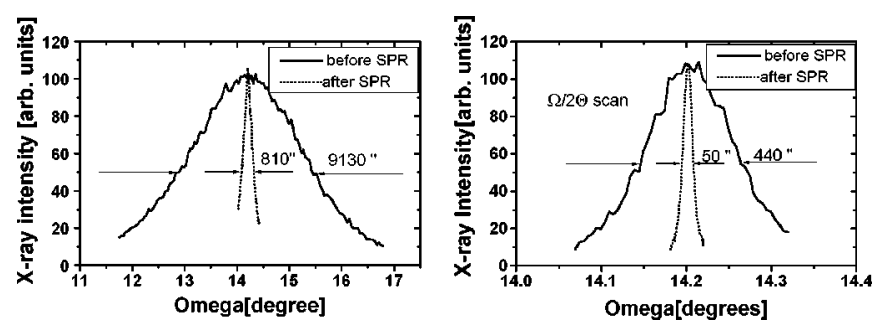

FIG. $4 . \Omega$ and $\Omega / 2 \Theta$ scan x-ray curves of the sample II366 before and after recrystallization process. The anneal temperature was $970{ }^{\circ} \mathrm{C}$ and the sulfur overpressure $10 \mathrm{~atm}$. The maximum intensities of the curves in the case of the recrystallized sample have been reduced to the maximum intensity of the initial curve, so that curve widths can be compared.

to AIP license or copyright, see http://apl.aip.org/apl/copyright.jsp 


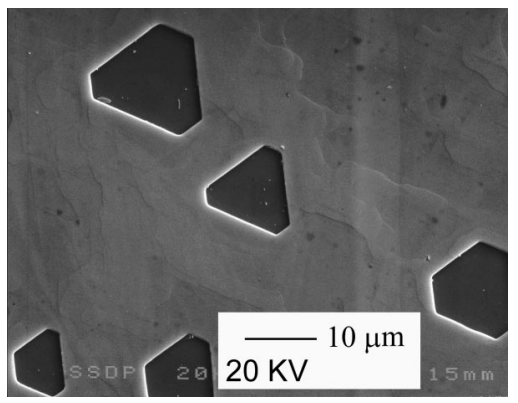

FIG. 5. Surface of the sample II391 recrystallized at $922^{\circ} \mathrm{C}$. The change in the surface morphology in form of hexagonal pits can be observed.

The role of the sulfur gas overpressure is manifold. First, the high sulfur overpressure greatly reduces zinc partial pressure, and thus prevents the evaporation of the $\mathrm{ZnS}$ film. Second, the sulfur-rich stoichiometry produces grain boundaries enriched by fast diffusing sulfur, which helps grain boundary migration. The important solubility of the chalcogen in the chalcogenides was accounted for to explain that in the case of bulk ZnSe successful recrystallization rates cannot be obtained under zinc overpressure. ${ }^{13}$ In addition, we hypothesize that sulfur might also play an important role at the $\mathrm{ZnS}$ / sapphire interface. The significant difference between recrystallization of thin films and bulk is the large mismatchrelated energy accumulated at the interface, which should reduce grain boundary mobilities. One possible route for the relaxation of this energy is a sulfur-rich $\mathrm{ZnS} /$ sapphire interface. Sulfur-terminated sapphire surface might change into a "quasi van der Waals" one, similar to the seleniumterminated GaAs surface. ${ }^{18}$ Weaker interface bonding could change interface structure, assist boundary migration and provide compliancy to the sapphire substrate.

In conclusion, $\mathrm{ZnS}$ thin films on sapphire were recrystallized, by annealing at temperatures above $900{ }^{\circ} \mathrm{C}$ at sulfur overpressure of $10 \mathrm{~atm}$. The structural properties of samples-quantified in terms of widths of x-ray $\Omega$ and $\Omega / 2 \Theta$ scans - significantly improved, indicating more than a tenfold reduction in tilting and excellent crystallinity. The electron channeling patterns confirmed good structural quality of the films. Surfaces of most recrystallized samples re- mained smooth, which is important for device-related applications. On some samples recrystallized at elevated temperatures the formation of hexagonal pits was observed and associated with the annihilation of a large number of defects initially occupying excess crystalline volume. The role of sulfur was discussed, both in preventing film evaporation and increasing boundary migration and providing compliancy to sapphire substrate.

One of the authors (Z.Z.B.) is indebted to Professor W. L. Johnson for helpful discussions. This work was supported by the Advanced Research Project Agency, and monitored by the Office of Naval Research under Grant No. N0001492-J-1845.

${ }^{1}$ S. Yamaga, Physica B 185, 500 (1993).

${ }^{2}$ T. Yokogawa and T. Narusawa, Appl. Phys. Lett. 61, 291 (1992).

${ }^{3}$ J. M. Depuydt, M. A. Haase, S. Guha, J. Qiu, H. Cheng, B. J. Wu, G. E. Hofler, G. Meishaugen, and M. S. Hagedorn et al., J. Cryst. Growth 138, 667 (1994).

${ }^{4}$ C. N. King, J. Vac. Sci. Technol. A 14, 1729 (1996).

${ }^{5}$ Y. Sato, N. Takahashi, and S. Sato, Jpn. J. Appl. Phys., Part 2 35, L838 (1996).

${ }^{6}$ J. O. McCaldin, M. W. Wang, and T. C. McGill, J. Cryst. Growth 159, 502 (1996).

${ }^{7}$ E. C. Piquette, Z. Z. Bandić, J. O. McCaldin, and T. C. McGill, J. Vac. Sci. Technol. B 15, 1148 (1997).

${ }^{8}$ J. E. Ayers, S. K. Ghandhi, and L. J. Schowalter, J. Cryst. Growth 113, 430 (1991).

${ }^{9}$ J. Petruzzello, K. W. Haberern, S. P. Herko, T. Marshall, J. M. Gaines, S. Guha, G. D. Uren, and G. M. Haugen, J. Cryst. Growth 159, 573 (1996).

${ }^{10}$ Physics and Chemistry of II-VI Compounds, edited by M. Aven and J. S. Prener (Wiley, New York, 1967).

${ }^{11}$ F. J. Humphreys and M. Hatherly, Recrystallization and Related Annealing Phenomena (Elsevier, New York, 1995).

${ }^{12}$ See for example M. J. Brau and R. A. Reynolds, US Patent No. 3, 849, 205 (1974).

${ }^{13}$ R. Triboulet, J. O. Ndap, A. Tromson-Carli, P. Lemasson, C. Morhain, and G. Neu, J. Cryst. Growth 159, 156 (1996).

${ }^{14}$ R. Triboulet, J. O. Ndap, A. El Mokri, A. Tromson-Carli, and A. Zozime, J. Phys. IV 5, 141 (1995).

${ }^{15}$ E. Tournie, C. Morhain, G. Neu, M. Laügt, C. Ongaretto, J. P. Faurie, R. Triboulet, and J. O. Ndap, J. Appl. Phys. 80, 2983 (1996).

${ }^{16}$ M. Meshkinpur, M. S. Goorsky, G. Chu, D. C. Streit, T. R. Block, and M. Wojtowitz, Appl. Phys. Lett. 66, 748 (1995).

${ }^{17}$ F. C. Brown, The Physics of Solids-Ionic Crystals, Lattice Vibrations and Imperfections (Benjamin, New York, 1967).

${ }^{18}$ A. Koma, Prog. Cryst. Growth Charact. Mater. 30, 129 (1995). 